\title{
Reverse Translation in Parkinson Disease
}

\author{
Roger L. Albin ${ }^{1-4}$ and Kirk A. Frey ${ }^{2,5}$ \\ ${ }^{I}$ Neurology Service \& GRECC, VAAAHS, Ann Arbor, Michigan; ${ }^{2}$ Department of Neurology, University of Michigan, Ann Arbor, \\ Michigan; ${ }^{3}$ University of Michigan Morris K. Udall Center, Ann Arbor, Michigan; ${ }^{4}$ Michigan Alzheimer Disease Center, Ann Arbor, \\ Michigan; and ${ }^{5}$ Division of Nuclear Medicine, Department of Radiology, University of Michigan, Ann Arbor, Michigan
}

$\mathbf{I}_{n}$ this issue of The Journal of Nuclear Medicine, Peng et al. describe an interesting experiment aimed at extending the use of a biomarker identified in clinical research on Parkinson disease (PD) to preclinical research (1). Although translational research is conceived commonly as flowing from basic bench research to preclinical model experiments to clinical experiments to application, this experiment describes reverse translation; a thoughtful exploration of clinical research results in a preclinical model. Biomarker is a much used, and perhaps much abused, term that is applied to a wide variety of measurements. Biomarkers (biologic markers) are generally conceived as quantifiable and objectively measured descriptors of some biologic state. In the context of pathobiology, biomarkers can measure normal physiology, pathologic processes, or the effects of interventions.

Biomarker-related research is increasingly prevalent in many clinical neuroscience disciplines. The considerable interest in biomarkers is driven by the apparently poor record of translating promising bench research into successful treatments. The National Institutes of Health is increasingly involved in explicitly translational

\section{See page 1591}

research and increasingly concerned with the meager yield of translational research. National Institutes of Health attempts to improve translational research include enhanced emphasis on rigor and transparency of translational research (https://www.nih.gov/researchtraining/rigor-reproducibility) and the implementation of grant mechanisms aimed explicitly at translational research (http://www.ninds.nih. gov/funding/areas/translational_research/). These efforts to improve translational research increasingly involve biomarker development, validation, and application.

There are many reasons for translational research failures. These include incomplete understanding of pathogenic processes, preclinical models with poor or unknown validity, preclinical experiments with poor experimental design and statistical planning, clinical studies with poorly chosen endpoints, clinical studies enrolling heterogeneous populations, and many others. Well-chosen biomarkers are suggested

Received Mar. 25, 2016; revision accepted Mar. 30, 2016.

For correspondence or reprints contact: Roger L. Albin, 5023 BSRB, 109

Zina Pitcher Place, Ann Arbor, MI 48109-2200.

E-mail: ralbin@umich.edu

Published online May 5, 2016.

COPYRIGHT (C) 2016 by the Society of Nuclear Medicine and Molecular Imaging, Inc.

DOI: $10.2967 /$ jnumed.116.175257 to be solutions to some of these problems. Trait biomarkers, serving to accurately differentiate subject subgroups, may address the problem of enrolling heterogeneous subjects in trials. In recent trials of anti-amyloid passive immunotherapy for Alzheimer disease (AD), for example, a significant fraction of enrolled subjects did not have $\mathrm{AD}$ as assessed by amyloid PET imaging (2). An ongoing, large study of anti-amyloid passive immunotherapy in presymptomatic $\mathrm{AD}$, the $\mathrm{A} 4$ trial, attempts to mitigate this problem through the use of amyloid PET as a gatekeeper for trial entry (https://www. nia.nih.gov/alzheimers/a4-study).

Another use of biomarkers is as a measure of disease activity (state biomarkers). These may be measures of the primary pathogenic process or a closely correlated epiphenomenon. The use of erythrocyte sedimentation rate in inflammatory disorders to adjust therapies is an example of the latter. In fields in which clinical rating scales have been the primary measurement instruments, appropriate biomarkers may provide more sensitive or more statistically robust measures of disease activity to measure intervention efficacy. These methods may allow smaller, faster trials, and may even serve as surrogates for recognized clinical endpoints. Much of the recent success in the development of multiple sclerosis therapies is attributed to MRI biomarker methods quantifying disease activity. Recent natural history studies suggest that MRI morphometry may be a useful method for evaluation of potential therapies in premanifest Huntington disease, in which trials using conventional clinical measures may not be feasible $(3,4)$.

Another important potential use of biomarkers in clinical research is target engagement. Is a potential therapy actually acting through the hypothesized mechanism or affecting the pathogenic process? Relatively small clinical experiments with suitable biomarkers demonstrating lack of target engagement would prevent wasted effort and resources on fruitless, large-scale clinical studies. Different biomarkers could be used to demonstrate target engagement in different ways. Target engagement of a drug thought to act at a specific receptor subtype, for example, can be assessed in competition studies with PET ligands for that receptor subtype. As shown by the example of multiple sclerosis, a biomarker of disease activity can be used in smaller, initial studies to provide evidence of efficacy justifying larger trials with clinical endpoints. A potentially powerful approach to target engagement is to use a biomarker that provides analogous information in both preclinical and clinical experiments. This approach would definitely enhance the utility of preclinical models.

Peng et al. evaluated a potential biomarker of circuit dysfunction in PD based on ${ }^{18} \mathrm{~F}$-FDG PET imaging. Using a form of principal component analysis, this group previously demonstrated a statistically robust pattern of brain metabolic changes in PD-the Parkinson disease-related pattern (PDRP) (5). This pattern may differentiate PD from closely related neurodegenerative disorders (6). The pattern 
tends to normalize with dopamine replacement therapy or with subthalamic nucleus gene therapy, indicating that it reflects the broad alterations in transregional circuit activities that follow significant nigrostriatal dopaminergic denervation $(7,8)$. This group subsequently demonstrated the existence of a closely analogous pattern in the wellestablished 1-methyl-4-phenyl-1,2,3,6-tetrahydropyridine nonhuman primate model of parkinsonism - the parkinsonism-related pattern (PRP) (9). In this experiment, Peng et al. show that the PRP tends to normalize after intrastriatal grafting of human retinal pigment epithelial cells (hRPE), a dopamine-secreting cell population. Because hRPE grafts produced significant improvement in motor function, and taking into account the human data, PRP and PDRP are plausible target engagement biomarkers. A target engagement biomarker applicable in both preclinical and clinical research is an attractive prospect. There is considerable interest in nondopaminergic pharmacologic or other interventions that would favorably modulate the transregional circuit dysfunctions of PD. These imaging measures could be used in pilot studies as decision milestones for initiating larger, definitive studies in either preclinical or clinical experiments.

This experiment also demonstrates some of the potential limitations of biomarkers. The response of a biomarker to an intervention could be misleading. Intrastriatal implantation of hRPE cells for PD was the subject of a well-performed human trial (10). This trial returned a clear negative result, largely because of a significant placebo response in the sham surgery arm of the trial. Peng et al. recognize this fact and argue that an advantage of their nonhuman primate model is that the animals don't exhibit a placebo response. This begs the question, however, of whether the PDRP would be a useful target engagement biomarker. PDRP normalization could be associated with placebo responses. This result would be fascinating but would obviate the utility of the PDBP as a target engagement biomarker. The lack of a placebo response in 1-methyl-4-phenyl-1,2,3,6-tetrahydropyridine-lesioned nonhuman primates is, in fact, a limitation of the model.

A target engagement biomarker is useful in proportion to the importance of the target. Recent data on incident PD from the community-based CamPaIGN study indicate that at 10-y follow-up, three fourths of PD patients have either disabling cognitive impairment or postural stability impairments (11). These features of PD are largely unresponsive to treatments, dopamine replacement, or deep brain stimulation, aimed at correcting the circuit dysfunctions resulting from nigrostriatal dopaminergic denervation. Given that the PDRP and PRP are probable biomarkers of these circuit dysfunctions, the PDRP and PRP are unlikely to be useful target engagement biomarkers for assessing interventions aimed at the most pressing problems in PD clinical care.

Whatever the eventual utility of the PDRP and PRP in clinical and preclinical research in $\mathrm{PD}$, Peng et al. deserve considerable credit for attempting to extend the biomarker concept rigorously in preclinical research. This general approach may ultimately enhance preclinical research in PD and other areas.

\section{DISCLOSURE}

This work was supported by R56082941 and P50NS091856. No other potential conflict of interest relevant to this article was reported.

\section{REFERENCES}

1. Peng S, Ma Y, Flores J, et al. Modulation of abnormal metabolic brain networks by experimental therapies in a nonhuman primate model of Parkinson disease: an application to human retinal pigment epithelial cell implantation. J Nucl Med. 2016;57:1591-1598.

2. Salloway S, Sperling R, Fox NC, et al. Two phase 3 trials of bapineuzumab in mild-to-moderate Alzheimer's disease. N Engl J Med. 2014;370:322-333.

3. Paulsen JS, Long JD, Johnson HJJ, et al. Clinical and biomarker changes in premanifest Huntington disease show trial feasibility: a decade of the PREDICTHD study. Front Aging Neurosci. 2014;6:78.

4. Tabrizi SJ, Scahill RI, Owen G, et al. TRACK-HD Investigators. Predictors of phenotypic progression and disease onset in premanifest and early-stage Huntington's disease in the TRACK-HD study: analysis of 36-month observational data. Lancet Neurol. 2013;12:637-649.

5. Ma Y, Tang C, Spetsieris PG, Dhawan V, Eidelberg D. Abnormal metabolic network activity in Parkinson's disease: test-retest reproducibility. J Cereb Blood Flow Metab. 2007;27:597-605.

6. Tripathi M, Tang CC, Feigin A, et al. Automated differential diagnosis of early parkinsonism using metabolic brain networks: a validation study. $J$ Nucl Med. 2016;57:60-66.

7. Asanuma K, Tang C, Ma Y, et al. Network modulation in the treatment of Parkinson's disease. Brain. 2006;129:2667-2678.

8. Feigin A, Kaplitt MG, Tang C, et al. Modulation of metabolic brain networks after subthalamic gene therapy for Parkinson's disease. Proc Natl Acad Sci USA. 2007;104:19559-19564.

9. Ma Y, Peng S, Spetsieris PG, Sossi V, Eidelberg D, Doudet DJ. Abnormal metabolic brain networks in a nonhuman primate model of parkinsonism. J Cereb Blood Flow Metab. 2012;32:633-642.

10. Gross RE, Watts RL, Hauser RA, et al. Intrastriatal transplantation of microcarrier-bound human retinal pigment epithelial cells versus sham surgery in patients with advanced Parkinson's disease: a double-blind, randomised, controlled trial. Lancet Neurol. 2011;10:509-519.

11. Williams-Gray CH, Mason SL, Evans JR, et al. The CamPaIGN study of Parkinson's disease: 10-year outlook in an incident population-based cohort. J Neurol Neurosurg Psychiatry. 2013;84:1258-1264. 